\title{
An Inquiry into Belonging, Connection, and Identity
}

\author{
Marta Callizo Giró \\ The MIECAT Institute, Australia
}

Keywords: arts as research, connection, identity, belonging, voluntary migrants

\section{Involve me and I will understand}

In this article I recount my experience of using arts as research in the context of my Master's research project. An Inquiry into Belonging, Connection, and Identity stemmed from the wish to gain a deeper understanding on the experience of voluntary migrants who settle away from their original homeland.

Comprehending our sense of belonging, connection and identity is a complex undertaking, for one might find that when we try to put it into words the narrative becomes entangled and hazy. Words fall short to explain such complex matters. For this reason, I felt drawn to exploring the topic through experiential inquiries, placing greater attention on felt sensing and multi-modal representations.

The first block of the project was dedicated to running a process of inquiry with three volunteer participants and myself in the roles of participant and researcher. Much of the data was co-created during this first part.

The second block of the project rooted its structure in employing the arts as research, using my experience as an artist to deepen my understandings of the data generated to date and to attain new approximations to meaning (findings). Throughout this stage of experiential exploration, I worked with a range of different creative modalities, including filmmaking, painting, knitting, and body gesture. In my role of art-maker/researcher I established an intersubjective dialogue with the art materials, and I stayed very present to the content that emerged during the process. This direct form of art-making as a primary mode of systematic inquiry provided me with many opportunities to embody some of the content that had emerged during the inquiries with the participants, generating a more direct understanding of what we had come to know thus far. Responding creatively not only helped me to make sense of it; the method also turned into content and helped generate experiential understandings and new data.

I started this process of iterative reflexivity by reducing, clustering, and synthesising all the data. I obtained five clusters that were each further reduced to a creative synthesis. Here I will show examples of my exploration of three of these clusters.

\section{"New World"}

The first cluster to work with was "New World". I chose to make a short animation film to represent the transition between one's original homeland and the new environment. I started by making a rough claymation, as a sketch, to have a sense for what I could make later on in a digital medium. 

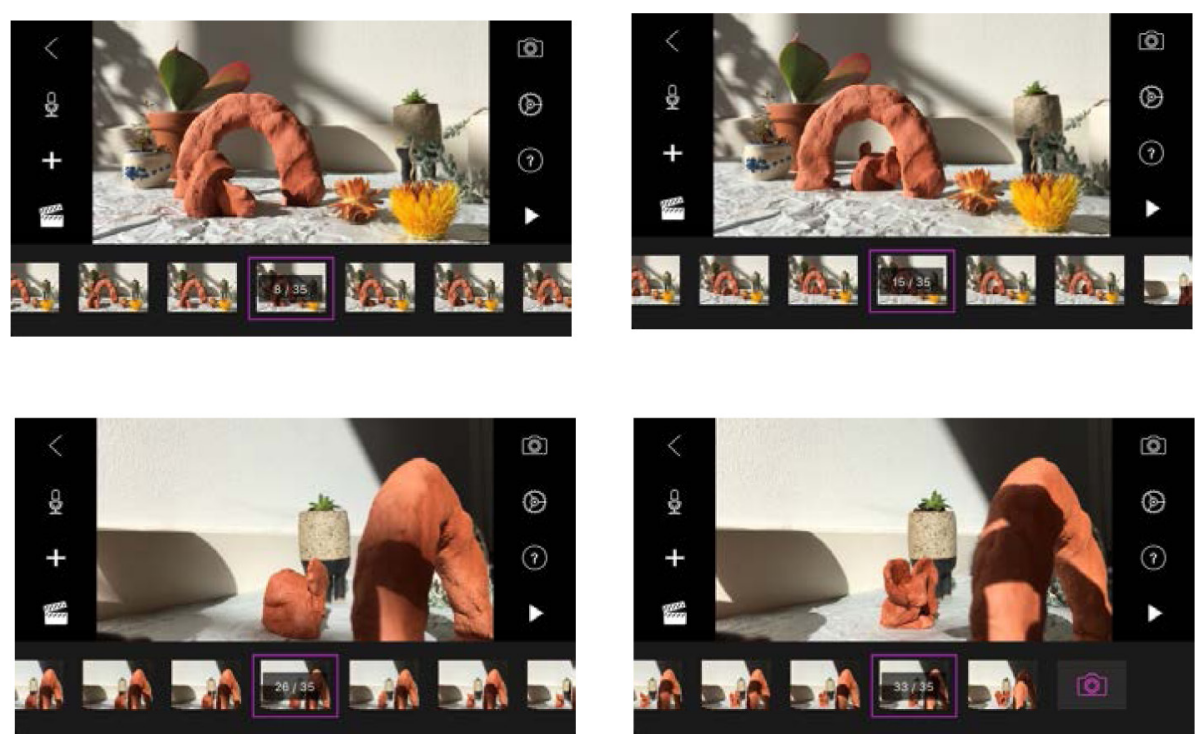

FIGURE 1 | Marta Callizo, Stills from “New World", 2017, (Claymation) See video at https://vimeo.com/260503574 (password: MIECAT18)

Working with clay became a pivotal point in the development of the film. As soon as I started working with my hands, the material informed the qualities of the little creature and directed its behaviour. McNiff (2011) writes about the need that some may have to "begin their work by simply making spontaneous expressions with the goal of seeing what will emerge and how the free movements can give shape to various themes and needs that may be circulating unseen in a person's consciousness" (p. 392). In this case establishing a dialogue with the clay allowed me to connect with the felt sense of an abstract representation of adaptation.

Following this Claymation sketch, I moved onto making a short digital animation film. The new media allowed for new forms and a deeper level of abstraction and the process of learning how to make the film became a re-enactment of moving to a country with an unfamiliar culture and language.

\section{(Ways of ) Coming Together}

The next cluster to respond to was "(Ways of) Coming Together", with body movement and voice. This response emerged as the result of attuning deeply to the felt sense and allowing the body to take the lead.
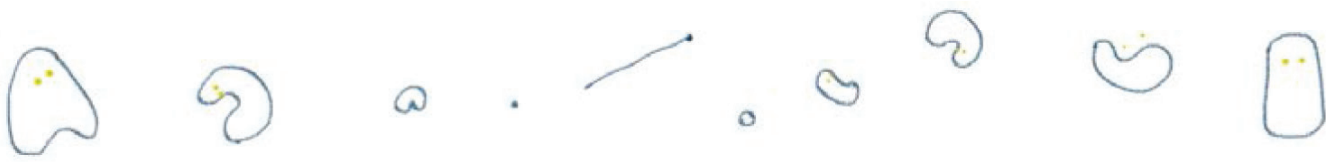

FIGURE 2 | Marta Callizo, Sequence from "New World", 2017, (digital media) See video at https://vimeo.com/260499130 (password: MIECAT18) 

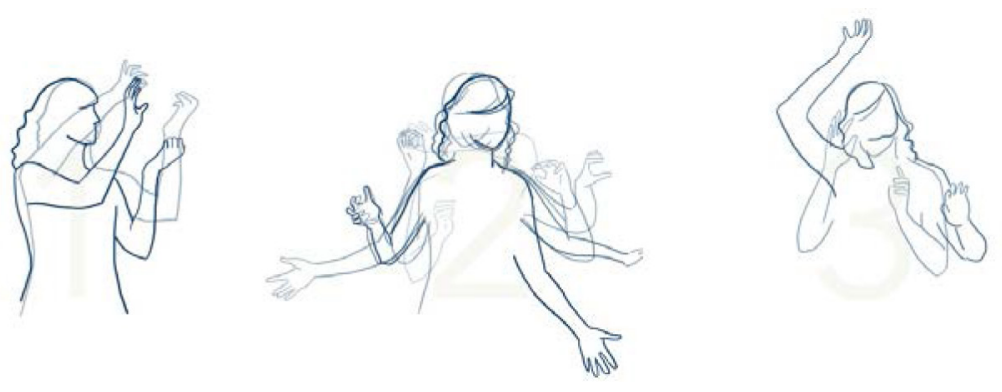

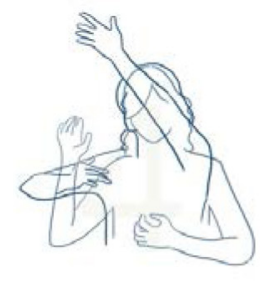

diversity

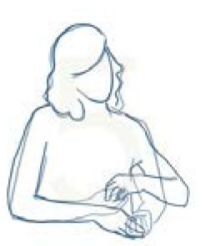

coming together

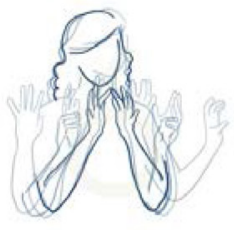

touch

FIGURE 3 | Marta Callizo, Gesture “(Ways of) Coming Together”, 2017, (digital image)

I repeated the sequence a few times, recorded it and wrote a phenomenological description. Then I reduced some key words and phrases extracted from the description to:

Hands move fast

Collecting

Moving fast

Scooping, pushing

\section{Diversity Coming together}

Arms slow down

Picking delicately

Holding

Bending forward

\section{Touch}

Working on this creative synthesis led me to the statement: The two parts come together after slowing down. They establish contact very gently.

\section{Demiurge}

A third cluster was reduced to the word "Demiurge", referring to Plato's artisan-like figure responsible for the fashioning and maintenance of the physical universe. I felt drawn to represent this synthesis in the form of a knitted hoop. I wanted it to be an item that reflected continuity, with no beginning or end. This informed the choice for the 


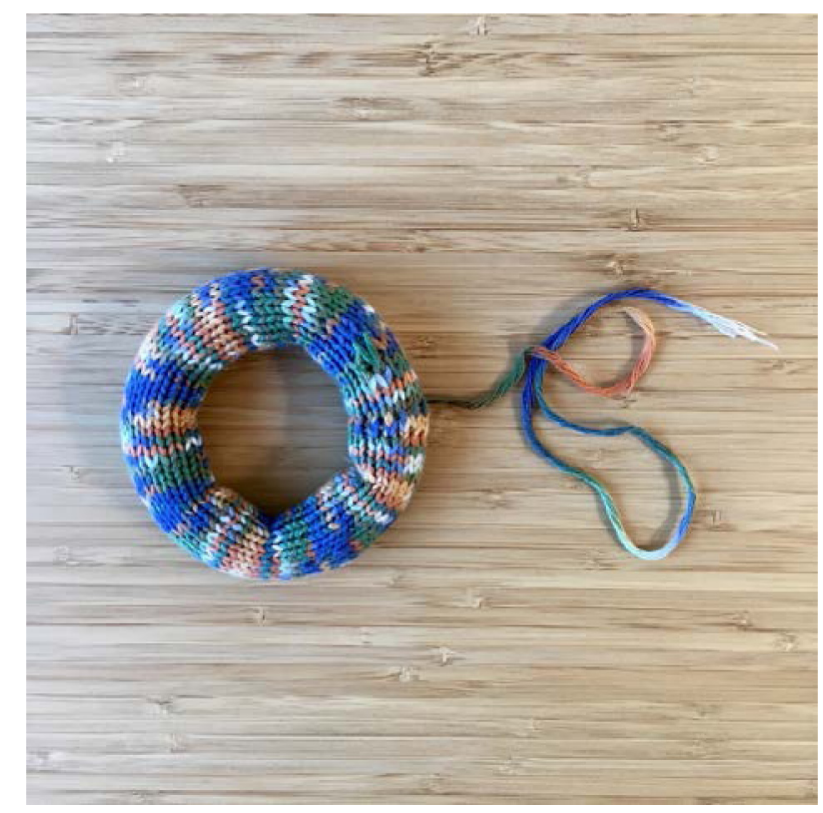

FIGURE 4 | Marta Callizo, ISR for "Demiurge" (final iteration), 2017, (knitted piece)

circular shape. It also had to be three-dimensional and constituted by the sum of multiple small elements (stitches). The addition of stitches would create the whole. These elements had to reflect diversity - expressed by shades of different colours - but also show rhythm and structure.

In the process of knitting the hoop I made mistakes and its skin became irregular, imperfect, the seam visible. It started from a definite concept, but the result was slightly different. It involved learning, trial and error, testing materials, and time constraints. It started as a challenge that required my full attention at first, and that allowed me to drift away and relax in the flow of the making later. In the process of responding to this cluster I enacted much of the content that emerged with the participants, reinforcing the finding that there is a strong bond between being creative and the sense of belonging.

\section{Wearing the artist hat}

These are just a few examples of how using the arts in research provided a platform for much deeper understandings and new findings. After responding to each cluster with five different modalities I came to observe that there were three main subjects emerging in parallel and I further developed them with the support of references to literature. Engaging with the data and bracketing in my resonances to respond with new creative presentations was not always an easy task. I encountered many moments of hesitance in which I feared bringing too much of my involvement forward and consequently overwriting the data generated with the participants. However, after much consideration I understood that by this point I had become the vessel of the four voices and decided to 
trust that my intersubjective engagement with the materials during the process of artmaking was conveying not only my own resonances but also a synthesis of the new approximations to meaning that had been reached in the co-creative space between myself and each one of the participants.

\section{About the Author}

Marta Callizo Giró, MArch, MTAP (Research). Marta completed her Master's in Architecture in Barcelona and has been living in Melbourne and working in the field of architecture for more than a decade. For the past five years Marta has combined her architecture career with her training as a therapeutic arts practitioner at The MIECAT Institute, where she completed her Master's in Therapeutic Arts Practice and currently works as an academic staff member. Marta's main interests lie in creativity, identity and relational dynamics.

\section{Reference}

McNiff, S. (2011). Artistic expressions as primary modes of inquiry. British Journal of Guidance and Counselling, 39(5): 385-396. 